\title{
The phylogeny of Anophelinae revisited: inferences about the origin and classification of Anopheles (Diptera: Culicidae)
}

\author{
Ralph E. Harbach \& Ian J. Kitching \\ Department of Life Sciences, Natural History Museum, Cromwell Road, London SW7 5BD, \\ UK
}

Corresponding author: Ralph E. Harbach, Department of Life Sciences, Natural History Museum, Cromwell Road, London SW7 5BD, UK. E-mail: r.harbach@nhm.ac.uk

Ralph E. Harbach, Ian J. Kitching, Department of Life Sciences, Natural History Museum, Cromwell Road, London SW7 5BD, UK. E-mails: r.harbach@nhm.ac.uk; i.kitching@nhm.ac.uk

Short title: Phylogeny of Anophelinae 


\section{Abstract}

The evolution of anopheline mosquitoes (Culicidae: Anophelinae) has been the subject of speculation and study for decades, but a comprehensive phylogeny of these insects is far from complete. The results of phylogenetic studies based on morphological and molecular data sets are conspicuously ambiguous. Here we revisit the phylogenetic relationships of anopheline mosquitoes using state-of-the-art software and cladistic methods to analyse the data set of Harbach \& Kitching (2005). We present a refined interpretation of relationships based on analyses of a revised data set that includes an additional species. Implied weighting analyses were conducted with TNT with the concavity constant $K$ ranging from $1-33$. We determined the optimal $K$ value by summing the GC supports for each MPC and selected the tree with the highest support, $K=30$, as the preferred cladogram. We then collapsed the branches with GC support $<1$ to obtain the "best" topography of relationships. Genus Chagasia is the basalmost taxon of Anophelinae, and genus Anopheles is recovered as monophyletic but only if Anopheles implexus is excluded and genus Bironella is subordinated within it. The Afrotropical An. implexus is recovered as the sister to all other anophelines, and Christya Theobald, stat. nov., is elevated from synonymy with Anopheles Meigen as a subgenus to accommodate it. The other anophelines comprise two large clades. The first includes the reciprocally monophyletic subgenera Kerteszia + Nyssorhynchus; the second consists of subgenus Cellia as the sister to a heterogeneous clade that includes genus Bironella and subgenera Anopheles, Baimaia, Lophopodomyia and Stethomyia of genus Anopheles. The sister relationship of Cellia and the heterogeneous clade is lost when the branches with GC < 1 are collapsed. The monophyly and non-monophyly of the informal subordinate taxa of subgenera Nyssorhynchus, Cellia and Anopheles, and also evolutionary scenarios, are discussed in relation to previous studies. 


\section{Introduction}

Culicidae, mosquitoes, comprise a large and abundant group of 3,543-3,546 formally recongized species with distributions in temperate and tropical regions of the world. The species are classified in two subfamilies, Anophelinae (482 $\underline{485}$ species) and Culicinae $(3,061$ species). Subfamily Anophelinae comprises three genera: Anopheles Meigen (cosmopolitan, 469-472 species), Bironella Theobald (Australasian, 8 species) and Chagasia Cruz (Neotropical, 5 species) (http://mosquito-taxonomic-inventory.info/). Mosquitoes of these genera are known in the vernacular as "anophelines".

Anopheles has been the subject of more taxonomic research than any other genus of mosquitoes because it includes the species that transmit malarial and filarial parasites to humans. The majority of anopheline species belong to this genus, which comprises seven subgenera: Anopheles s.s. (cosmopolitan, $182 \underline{185}$ species), Baimaia (Oriental, 1 species), Cellia Theobald (Old World, 224 species), Kerteszia Theobald (Neotropical, 12 species), Lophopodomyia Antunes (Neotropical, 6 species), Nyssorhynchus Blanchard (Neotropical, 39 species) and Stethomyia Theobald (Neotropical, 5 species) (http://mosquito-taxonomicinventory.info/). The subgenera are based principally on the number and positions of specialized setae borne on the gonocoxites of the male genitalia (Christophers 1915; Reid 1968; Harbach \& Kitching 2005). The three largest subgenera, Anopheles, Cellia and Nyssorhynchus, are divided into hierarchical systems of informal taxonomic categories (Reid \& Knight 1961; Harbach 1994, 2004). Subgenus Anopheles is divided into two Sections based on the shape of the pupal trumpet (Reid \& Knight 1961) and subgenus Nyssorhynchus is divided into three Sections based on characters of the larvae, pupae and adults (Faran 1980; Harbach 1994). Subgenus Cellia and the Sections of subgenera Anopheles and Nyssorhynchus are divided into Series, the larger Series are divided into species Groups, and some Groups are further divided into Subgroups and species Complexes. It is generally 
assumed that each level of classification comprises a more or less natural assemblage of species based on morphological similarity. Genus Bironella includes three subgenera, but unlike the larger subgenera of Anopheles, they are not subdivided into informal group taxa. Genus Chagasia is a small homogenous group of species that is also not subdivided (Harbach \& Howard 2009).

Sallum et al. (2000) conducted the first phylogenetic study of Anophelinae, based on morphological traits. The results indicated that genus Anopheles is paraphyletic because it included genus Bironella. Subgenera Kerteszia, Nyssorhynchus, Cellia, Lophopodomyia and Stethomyia, along with genus Bironella, were found to be monophyletic taxa dispersed among various Series and species Groups of subgenus Anopheles. The Christya Series of subgenus Anopheles was placed with Kerteszia + Nyssorhynchus and this clade was sister to Cellia + all other anophelines except Chagasia.

Sallum et al. (2002) assessed anopheline relationships based on ribosomal (18S, 28S) and mitochondrial (COI, COII) DNA sequences for half of the species included in the analyses of Sallum et al. (2000). Although the results of the two studies cannot be compared directly, analyses of the available molecular data corroborated the paraphyly of genus Anopheles relative to Bironella, the sister-group relationship of Kerteszia and Nyssorhynchus, and the monophyly of subgenera Cellia, Lophopodomyia and Stethomyia and genus Bironella, which was recovered as the sister of Lophopodomyia rather than Stethomyia.

Harbach \& Kitching (2005) revised and expanded the data set of Sallum et al. (2000), including reinterpretation of certain homologies (especially the specialized setae of the male gonocoxites that diagnose the subgenera), revised coding of some characters, and addition of new data and two further taxa, to reassess the phylogeny of Anopheline. Parsimony analysis of the data set under implied weighting supported the monophyly of subgenera Cellia, Kerteszia and Nyssorhynchus, and the sister relationship of Kerteszia + Nyssorhynchus. 
Subgenus Anopheles was recovered as a polyphyletic lineage basal to a clade consisting of Cellia $+($ Kerteszia + Nyssorhynchus $)$. Bironella, Lophopodomyia and Stethomyia were nested within subgenus Anopheles, and subgenus Baimaia was recovered as the sister of Bironella + all other Anopheles. In contrast to the results of Sallum et al. (2000), Bironella and Stethomyia were recovered as monophyletic groups separate from subgenus Anopheles.

In summary, the phylogenetic studies conducted to date disclose the following principal conclusions about the phylogeny and classification of anopheline mosquitoes: (1) subfamily Anophelinae is a monophyletic lineage basal to all other Culicidae; (2) genus Chagasia is basal to the other anopheline taxa; (3) genus Anopheles is not demonstrably monophyletic with regard to genus Bironella and subgenera Lophopodomyia and Stethomyia; (4) subgenera Kerteszia, Nyssorhynchus and Cellia are each monophyletic (subgenus Baimaia is monobasic); (5) Kerteszia and Nyssorhynchus are sister taxa; and (6) the monophyly of the informal Sections and most Series of subgenera Anopheles, Cellia and Nyssorhynchus is doubtful (Harbach \& Kitching 1998; Sallum et al. 2000, 2002; Krzywinski et al. 2001a, b; Harbach \& Kitching 2005; Harbach 2007, 2013).

Harbach (2013) maintained that the preferred cladogram of Harbach \& Kitehing (2005: figs 2 and 3) is currently the best hypothesis of anopheline phylogeny because it is based on analyses of a greater number of taxa and morphological characters than all other published hypotheses. But is it really the best estimate of relationships? We thought so until The monophyly of the Cyclopeppteron Series of subgenus Anopheles has been in doubt since it was redefined by Reid \& Knight (1961) to include only An. annulipalpis Lynch Arribálzaga (known only from Argentina and Uruguay) and An. grabhamii Theobald (endemic to the West Indies). In 2014, we received a request from Gustavo Rossi (Consejo Nacional de Investigaciones Científicas y Técnicas, La Plata, Argentina) questioned the inclusion of An. annulipalpis Lynch Arribálzaga (known only from Argentina and Uruguay), 
along with An. grabhamii Theobald (endemic to the West Indies), in the Cycloleppteron Series (only two species) of subgenus Anopheles and asked us to re-run the 2005-data set of Harbach \& Kitching (2005) with the addition of character data for An. annulipalpis (An. grabhamii was already included). The morphology-based phylogenetic study of subgenus Anopheles conducted by Collucci \& Sallum (2007) had indicated that the two species are not closely related, and Gustavo wanted to confirm this based on cladistic analysis of a larger data set. We immediately realized that the data set should not be analysed using the same software and methods used in 2005. In our previous study, we analysed the data using implied weights, implemented by PIWE version 3.0 (for Windows) (Goloboff 1997), with the default value of the concavity constant, $K=3$. More recently, commencing with our collaborative study on the phylogeny of Aedini (Reinert et al. 2009), we conducted cladistic analyses using TNT version 1.1 (Willi Hennig Society Edition) (Goloboff et al. 2008), in which any value of $K$ can be applied, and exploring the results of analyses using a much broader range of $K$ values. Furthermore, we now agree subsequent to our 2005 paper, with Sereno (2007) that provided strong and logical arguments for separating the neomorphic and transformational elements of multistate characters should be coded separately (using “contingent coding”; Forey \& Kitching 2000), arguments that we find compelling, and : Econsequently, recoding of the multistate characters employed by Harbach \& Kitching (2005) became necessary. We also deemed it appropriate to apply more recently developed and stringent methods of assessing clade support. With this as background, we present here a refined interpretation of anopheline relationships based on analyses of a revised data set, with the inclusion of An. annulipalpis.

\section{Materials and methods}




\section{Morphology}

As indicated in the Introduction, we added An. annulipalpis to the data set of 66 anopheline species analysed by Harbach \& Kitching (2005: Appendix). The full data set of 69 taxa thus comprised an ingroup of 67 species of Anophelinae and two outgroup species from subfamily Culicinae: Aedeomyia (Aedeomyia) squamipennis (Lynch Arribálzaga) and Uranotaenia (Uranotaenia) lowii Theobald. The data set of Harbach \& Kitching (2005) included 46 compound multistate characters that required subdivision into one or more characters, resulting in an increase from 167 to 224 morphological characters (Appendix S1) coded from adults (55), male genitalia (35), fourth-instar larvae (95) and pupae (39). The coded data are provided in Appendix S2.

\section{Phylogenetic analyses}

Parsimony analyses were implemented with TNT version 1.1 (Willi Hennig Society Edition, August 2011) (Goloboff et al. 2008) using both equal weighting (EW) and implied weighting (IW) with values of the concavity constant, $K$, ranging from $1-33$. The value of $K$ indicates inversely the weighting "strength" applied, with low values weighting more strongly against homoplastic characters (measured as the number of extra steps required to fit the cladogram topology in question) and higher values weighting less strongly (Goloboff 1993). The individual character weights are summed to produce the overall "fit" and the most parsimonious cladogram (MPC) is that with the greatest fit. Heuristic searches were conducted using the new technology search options: sectorial searches, ratchet, tree drifting and tree fusing. For the ratchet, the up/downweighting probabilities were set to $5 \%$ and the number of replicates to 200 . The number of cycles of tree drifting was set to 50. All other search parameters remained at their default settings. Analyses were terminated once the MPC had been found 100 times. The maximum number of trees held was set to 10,000 . Multistate 
characters were treated as unordered and cladograms were rooted between Ad. squamipennis and the remaining taxa.

The relative support for each node was assessed using symmetric resampling, as implemented in TNT, recording the frequency differences, i.e. "Groups present / Contradicted" or GC values (Goloboff et al. 2003). This metric does not suffer from the frequency distortions seen in other methods that resample to assess group support, such as the bootstrap and jackknife, and which are particularly pernicious when applied to weighted data (Goloboff et al. 2003). The GC values assess the difference between the absolute frequency with which a clade is found in the resampled matrices and that in the most frequent alternative topology in which the clade is not recovered. The GC values range from 100 , where the clade is recovered in all resampled matrices, to -100, where an alternative arrangement is found in all resampled matrices (Goloboff et al. 2003). A zero value indicates that levels of support and contradiction are equal. Due to time constraints, we calculated GC values using the traditional search options, with 100,000 replicates and the default change probability, and searches constrained to use only those groups found in the MPC. We then summed the GC supports across all groups on each MPC and used this as the optimality criterion to select the best topology and its associated value of $K$ (Goloboff et al. 2003; González-Santillán \& Prendini 2015). Cladograms were prepared and morphological character mappings investigated using WinClada ver. 1.00.08 (Nixon 1999-2002).

\section{Results}

Each analysis produced only a single MPC, except for $K=3,4$ and 8 , where eight, three and two MPCs, respectively, were found. In contrast to the study of González-Santillán \& Prendini (2015: fig. 7), which found unimodal distributions of summed GC ( $\sum$ GC) values, each with a single clear maximum value, our results (Fig. 1) show three peaks of $\sum \mathrm{GC}$ at $K$ 
values of 3,8 and $30-33$, of which the highest is that for $K=30 / 32 / 33\left(\sum \mathrm{GC}=1837\right)$. However, as the $\sum \mathrm{GC}$ for $K=31$ was slightly lower at 1835 , we checked whether this decrease was simply a stochastic artefact. We replicated the GC calculations a further nine times for $K=30$ and $K=31$. The $K=30$ tree yielded $\sum$ GC values ranging from $1835-1837$ (average $=1836.5$ ) and the $K=31$ tree yielded $\sum \mathrm{GC}$ values ranging from $1834-1838$ (average $=1836.2$ ). There is thus clearly a stochastic element to the $\sum \mathrm{GC}$ calculations due to the pseudorandom nature of the resampling procedure, and so we also repeated the procedure for the two MPCs found with $K=8$, which had yielded the next highest $\sum$ GC (1830). Tree 0 yielded $\sum$ GC values ranging from $1819-1825($ average $=1822.1)$ and tree 1 yielded $\sum$ GC values ranging from $1825-1830$ (average $=1826.9)$. The highest value for $K=8(1830)$ was well separated from the lowest $\sum \mathrm{GC}$ for $K=30 / 31$ (1835), confirming our choice of the $K=$ 30-33 topology as our preferred pattern of relationships among anophelines.

The MPC for $K=30-33$ is shown in Figure 2. As demonstrated in previous studies (see Introduction), genus Chagasia is the basalmost taxon of Anophelinae. Genus Anopheles is recovered as monophyletic but only if genus Bironella is subordinated within it (see below). Anopheles (Ano.) implexus is placed as the sister to all other anopheline taxa, which fall into two large clades. The first comprises the reciprocally monophyletic subgenera Kerteszia and Nyssorhynchus. Within the second, subgenus Cellia is recovered as monophyletic and sister to the remaining taxa. The first to branch off within this latter clade is the representative of subgenus Lophopodomyia, followed by a grade comprising members of subgenus Anopheles. However, within the largest subclade of this latter group, subgenera Stethomyia and Baimaia and genus Bironella are arrayed in a terminal clade.

However, the GC supports also suggest that some groups are very poorly supported or unsupported. Collapsing those groups with GC $<1$ on any one of the 10 replicates of the GC calculations gave the topology shown in Figure 3 (maximum GC values $>0$ are shown, so the 
$\sum \mathrm{GC}$ of the figure is greater than 1837). The GC values of six of the remaining branches varied among the replicates but only by (a decrease of) 1 and never all at once in any one replicate. There is some loss of resolution within subgenera Nyssorhynchus and Cellia, but they each remain monophyletic. The sister-group relationship between Cellia and the clade comprised of the remaining taxa is also lost with the formation of a three-way polytomy. Within the third clade, the sister-group relationship between subgenus Lophopodomyia and the remaining taxa is lost within a basal eleven-way polytomy, as is also much of that within subgenus Anopheles. The subclade comprising An. (Ano.) punctipennis to An. (Ano.) sinensis has some loss of resolution but remains monophyletic. Most importantly, however, all the relationships within the clade comprising An. aitkenii $+($ An. corethroides $+($ Stethomyia + $($ Baimaia + Bironella))) survive.

\section{Discussion}

The monobasic Christya Series includes only the African species An. implexus. The $\underline{\text { Whereas }}$

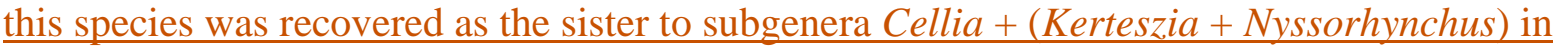
our previous study (Fig. S2), the results of the present analyses shown in Fig. 3 suggest that An. implexus it is sister to all Anophelinae except genus Chagasia, although the support for the latter group $(\mathrm{GC}=2)$ is weak. Reid \& Knight (1961) noted that Anopheles implexus shows-shares a number of affinities-features with Chagasia, including speckled legs, tarsi with unusually broad basal pale bands and a simple pupal trumpet. The basal position of the tarsal pale bands in particular is unusual for an anopheline and more like eertain-some species of subfamily Culicinae. These characters indicate that $A n$. implexus is a primitive member of genus Anopheles. Reid \& Knight (1961)also observed that An. implexus shows affinities with species of the Anopheles Series of subgenus Anopheles as well as groups of the Laticorn Section, but also suggested a relationship with subgenus Nyssorhynchus suggesting a 
somewhat intermediate position within the subgenus. In contrast, An. implexus and two species of the Arribalzagia Series comprised a terminal clade within a polyphyletic Myzorhynchus Series in the phylogeny of subgenus Anopheles recovered in the morphologybased study of Collucci \& Sallum (2007). Thus, the phylogenetic relationships of An. implexus are rather ambiguous. The results of the present study do not support a close affinity with subgenus Anopheles, and therefore not with species of the Anopheles and Myzorhynchus Series (Fig. 3). Features of the parabasal setae of the mate gonocoxitethe male genitalia seem to indicate a closer relationship with other taxa. The gonocoxite of $A n$. implexus bears a single parabasal seta that arises from an elongate prominence, whereas most species of subgenus Anopheles have multiple parabasal setae borne on a small protuberance. Exceptions includeThere are exceptions, however. An.Anopheles algeriensis and An. corethroides, the latter which is recovered as sister to a terminal clade comprised of Stethomyia $+($ Baimaia + Bironella $)(\text { Fig. 3) })_{2}$-both have a single parabasal seta and that of the former species is borne on an elongate prominence. Another distinctive feature of $A n$. implexus is the presence of posterolateral tufts of piliform scales on abdominal tergum IV, a feature that is shared with An. oiketorakras and An. nimbus of the Neotropical subgenera Lophopodomyia and Stethomyia, respectively, both of which appear to be more closely related to subgenus Anopheles than to An. implexus (Figs 2, 3). The scale-tufts are usually absent in species of subgenus Anopheles, and are spatulate rather than piliform when present. Larval seta 1-M of An. implexus is unique in having a long and broad rachis. The rachis is short or long and narrow in the other anopheline species included in the analyses. Somewhat similar to the results reported here, Sallum et al. (2000) found that a clade comprised of An. implexus $+($ Kerteszia + Nyssorhynchus $)$ was the earliest lineage of genus Anopheles. The relationship, however, was very weakly supported (bootstrap $<50 \%$; Bremer support 2). Reid \& Knight (1961) suggested a relationship between An. implexus and subgenus Nyssorhynchus 
based on the mutual occurrence of extensive pale scaling on the hindtarsi of adults and the unserrated leaflets of the abdominal palmate setae of larvae. In addition to the characters mentioned above, Wilkerson \& Peyton (1990) found that the wing of An. implexus is distinctive in having both apical and preapical pale spots, and noted that the presence of speckled legs, prominent abdominal scale tufts and pale hindtarsomeres-suggested that $A n$. implexus is "a possible ancestor of the New World species of the subgenera Nyssorhynchus, Kerteszia, Lophopodomyia, and the Arribalzagia Series".

\section{Subgenera Kerteszia and Nyssorhynchus}

Phylogenetic studies of anopheline mosquitoes based on both morphological and molecular data support the reciprocal monophyly and sister relationship of subgenera Kerteszia and Nyssorhynchus (Sallum et al. 2000, 2002; Collucci \& Sallum 2003; Harbach \& Kitching 2005). The sister relationship recovered in the collapsed tree (Fig. 3) is supported by six characters (Fig. S1), including three that are unique and not contradicted (63:1, accessory setae of gonocoxite present; 68:1, parabasal setae inserted on margin of gonocoxite; 77:1, specialized apical seta of ventral claspette present, spiniform and foliform). Relationships of the informal group taxa represented by the species that comprise the Nyssorhynchus clade are delineated in Figure 4. The monophyly of the subgenus is strongly supported by 12 characters (Fig. S1), two of which are unique and not contradicted (57:1, ventromesal connection of gonocoxite developed as a truncate process; 204:0, pupal seta 10-VI absent). However, the Myzorhynchella and Argyritarsis Sections are not monophyletic; the Myzorhynchella Section is paraphyletic relative to species of the Argyritarsis and Albimanus Sections. The Argyritarsis Series is polyphyletic and species of the Albimanus and Oswaldoi Series of the Albimanus Section fall in an unresolved clade together with a monophyletic Albitarsis Series of the Argyritarsis Section. The monophyly of the Albitarsis Series, however, is only weakly 
supported (GC = 8 or 9). Despite the more stringent assessment of clade support, it is worth noting that the same pattern of relationships was recovered as in our previous study (Fig. S3).

Bourke et al. (2010) performed a phylogenetic study of relationships among 21 species of subgenus Nyssorhynchus based on sequences for the mitochondrial ND6 and nuclear white genes. When the two genes were combined in a single analysis, the Myzorhynchella Section was recovered as a monophyletic group in a basal relationship to the Albimanus and Argyritarsis Sections, neither of which, in agreement with the results of the present study, were recovered as a monophyletic group. It is interesting to note that species of the Myzorhynchella Section were recovered as two separate clades in a polytomy with a third clade comprised of the other sections when the ND6 sequence data were analysed alone. The preponderance of evidence, gleaned from analyses of morphological and molecular data (Sallum et al. 2000, 2002; Harbach \& Kitching 2005; Bourke et al. 2010; present study), convincingly indicates that the current internal classification of subgenus Nyssorhynchus (Harbach 2013; http://mosquito-taxonomic-inventory.info/subgenusltemgtnyssorhynchusltemgt) is not based on natural relationships.

\section{Subgenus Cellia}

Phylogenetic analyses of morphological and molecular data have shownindicate that subgenus Cellia is a monophyletic group (Krzywinski et al. 2001a, b; Sallum et al. 2000, 2002; Harbach \& Kitching 2005; Mohanty et al. 2009; Wang et al. 2014; Norris \& Norris 2015). The Cellia clade shown in the MPC (Fig. 2) is supported by a combination of four characters, none of which are unique (Fig. S1). However, the clade is fairly well supported as indicated by a GC value of 47 (Fig. 3). It is interesting to note that in the analyses of Wang et al. (2014), Cellia was recovered as a monophyletic group based on D2 rDNA sequences of 28 species of Cellia and eight species of subgenus Anopheles, but was polyphyletic in a D3 
rDNA phylogeny that included sequences for 49 species of Cellia and the same eight species of subgenus Anopheles due to the exclusion of An. (Cellia) maculatus from the clade comprising subgenera Anopheles plus the remaining Cellia.

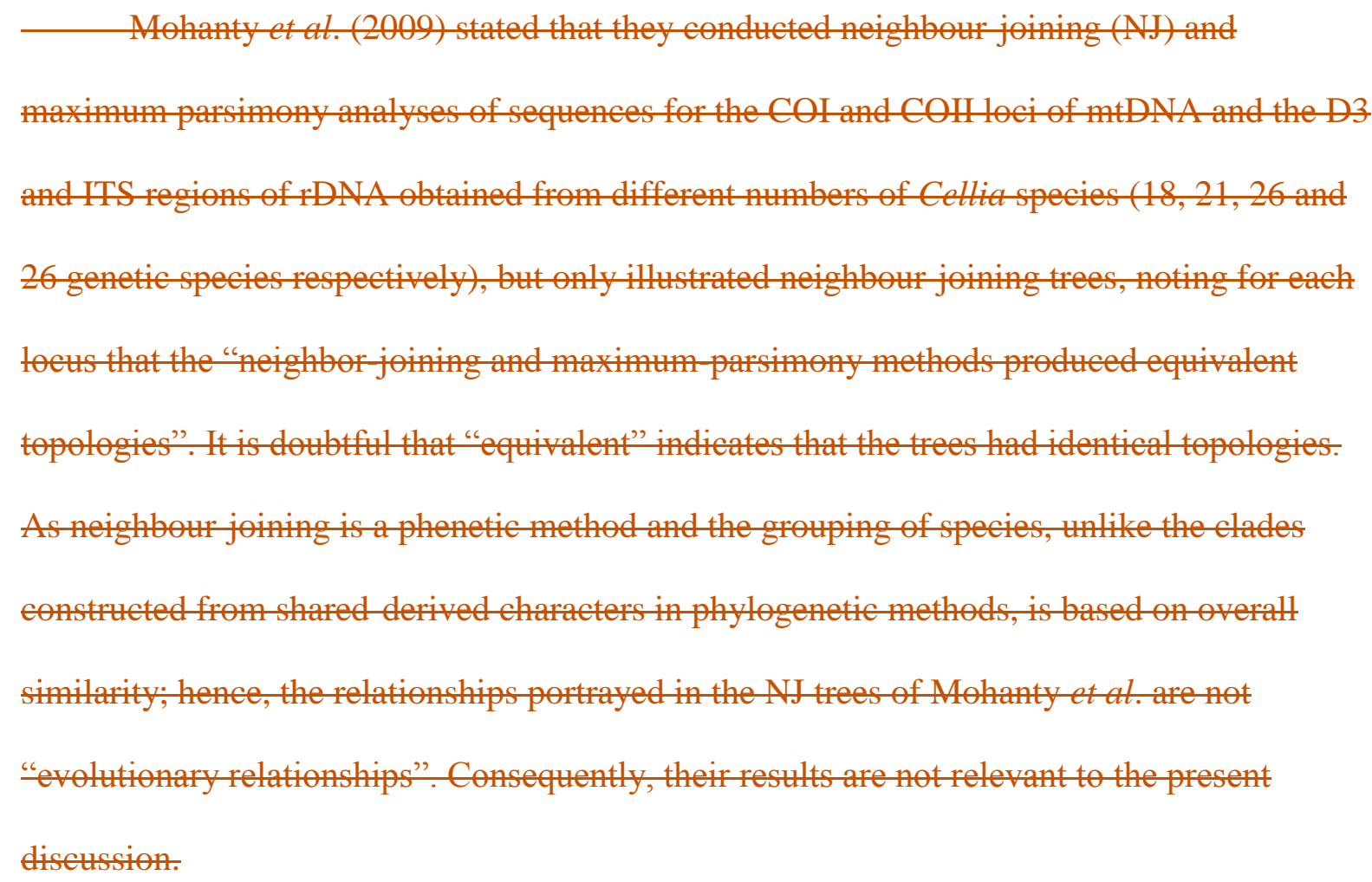

The internal classification of Cellia stems from the framework of Edwards (1932) and the revised and updated schemes of Grjebine (1966), Reid (1968), Gillies \& de Meillon (1968) and Harbach (1994). The subgenus is divided into six principal informal groups, the Cellia, Myzomyia, Neocellia, Neomyzomyia, Paramyzomyia and Pyretophorus Series, each of which has some or all of the included species classified in one or more species Groups. The subgenus includes 224 formally named species. The Cellia Series includes eight species, two of which form the subordinate Squamosus Group; the Myzomyia Series comprises 65 species, 17 unplaced and 48 divided between four species Groups; the Neocellia Series has 32 species, 15 unplaced and 17 classified in three species Groups; the Neomyzomyia Series includes 92 species, 18 unplaced and 74 divided between 10 species Groups; the Paramyzomyia Series has only six species split between two species Groups; and the 
Pyretophorus Series encompasses 21 species, 10 unplaced and 11 divided between three species Complexes (Harbach, 2013; http://mosquito-taxonomic-inventory.info/node/11370). The present analysis only included 21 species $(9 \%)$ of the subgenus $s_{2} \div$ two from the Cellia Series (one unplaced and one representing the Squamosus Group); six from the four species Groups of the Myzomyia Series; two fromrepresenting all the six series and all but two of the species groups of the Neocellia Series. (one unplaced and one representing one of the three species Groups); seven representing six of the 10 species Groups of the Neomyzomyia Series; two from the Paramyzomyia Series, representatives of the two species Groups; and two from the Pyretophorus Series, an unplaced species and a member of the Gambiae Complex.Despite the severely limited taxon representation of this and previous morphological (Sallum et al. 2000; Harbach \& Kitching 2005) and molecular studies (Sallum et al. 2002; Mohanty et al. 2009; Norris \& Norris 2015), it is obvious that a significant part of the current internal classification of Cellia does not reflect evolutionary relationships. $\underline{\text { In }}$ comparison with our previous study (Fig. S3), the relationships within Cellia recovered in the present study (Fig. 5) are more poorly resolved, but this, in part, is due to our now more stringent application of branch support. Whereas the Cellia, Myzomyia, Paramyzomyia and Pyretophorus Series were recovered previously as monophyletic groups (Fig. S3), The relationships of the informal group taxa delineated in Fig. 5 reveal that the Myzomyia, Neocellia, Neomyzomyia and Paramyzomyia Series are not monophyletic. The -only the Cellia Series now appears to be monophyletic (strongly supported, GC $=95$ ),, but $\underline{\text { However, }}$ the monophyly of the Pyretophorus Series is questionable due to the placement of An. vagus and An. gambiae as separate terminals within the eleven-way polytomy. These results mirror the results of Sallum et al. (2000), whose study included 61 fewer morphological characters for 64 of the 67 species included herein. As shown in many other studies (e.g. Sallum et al. 2007; Wang et al. 2014), the Funestus Group (Myzomyia Series) and the Leucosphyrus 
Group (Neomyzomyia Series) are monophyletic assemblages. It is noteworthy, however, that An. theileri Edwards of the Wellcomei Group of the Myzomyia Series was recovered within the Funestus Group in the maximum parsimony analyses of COI mtDNA and ITS2 rDNA sequence data conducted by Norris \& Norris (2015), indicating that the group may not be $\underline{\text { monophyletic. }}$

Sallum et al. (2002) conducted a molecular analysis of anopheline relationships based on ribosomal (18S, 28S) and mitochondrial (COI, COII) DNA sequences. Contrary to the findings reported here and by Sallum et al. (2000), the Myzomyia, Neocellia, Neomyzomyia and Pyretophorus Series were recovered as monophyletic groups based on analyses of the ribosomal and combined ribosomal and mitochondrial sequence data. Those results, however, eannot be construed to confirm the monophyly of the four series because significantly fewer taxa were included in the analyses. Whereas the present study and that of Sallum et al. (2000) included 21 species of subgenus Cellia, the molecular study of Sallum et al. (2002) only included sequence data for nine species. In addition to five of the species included here, $A n$. dirus, An. farauti (Neomyzomyia Series), An gambiae (Pyretophorus Series), An. funestus and An. minimus (Myzomyia Series), the molecular study included An. stephensi Liston (Neocellia Series, unplaced; substitution for An. superpictus), and three species, in addition to An. gambiae, of the Pyretophorus Series, An. arabiensis Patton (Gambiae Complex), An. sundaicus (Rodenwaldt) (Sundaicus Complex) and An. subpictus Grassi (Subpictus Complex). Despite the inclusion of three additional members of the Pyretophorus Series, in the absence of molecular data for An. vagus (Fig. 5), and probably also An. multicolor (sister to An. vagus in Fig. 2), the monophyly of the series must remain in doubt. Likewise, the Myzomyia and Neomyzomyia Series cannot be inferred to be monophyletic in the absence of data for those species included in the present study that cause these groups to be polyphyletic. Furthermore, the Neocellia Series cannot be regarded as being monophyletic based on the 
inclusion of only one species of the group (i.e. An. stephensi). It is interesting to note, however, that Bayesian analysis of D2 rDNA sequence of 28 species of Cellia (An. vagus absent) support the finding of Sallum et al. (2002), whereas their parsimony analysis of D3 rDNA sequence for 49 species of the subgenus (An. vagus included) only recovered the Neomyzomyia Series (four species) as monophyletic.

One is tempted to assume that $t$ The molecular phylogeny of Neafsey et al. (2015) based on protein sequences of 1,085 single-copy orthologs for seven species of the Pyretophorus Series - five species of the Gambiae Complex, An. christyi (unplaced) and An. epiroticus (Sundaicus Complex) - and the phylogeny of Anthony et al. (1999) based on morphological data for 10 species of the group, convincingly supports the monophyly of the series, - but in fact it is illogical to infer the monophyly of the entire series based on limited taxon sampling. The phylogeny of Neafsey et al. also included lineages that correspond to the Myzomyia Series (three species), Neocellia Series (two species) and Neomyzomyia Series (two species), but the probability that these clades accurately reflect a common ancestry for all species of each group is doubtful in view of the relationships recovered for significantly more species included in the morphological and other molecular studies conducted to date.

It is interesting to compare the relationships of the seven species of the Pyretophorus Series included in the study of Neafsey et al. (2015) with the relationships recovered among 10 species of the group in the morphological phylogenetic study of Anthony et al. (1999). The Oriental An. epiroticus was recovered as the basal taxon in the former study whereas the two Afrotropical species included in the latter study (An. christyi and An. gambiae) were basal and paraphyletic relative to the Oriental species, with An. christyi in the most basal position. Anopheles christyi was also recovered basal to the Afrotropical species (Gambiae Complex) in the phylogeny of Neafsey et al. (2015). 


\section{Subgenus Anopheles}

The clade comprising subgenus Anopheles in the MPC (Fig. 2) is supported by a combination of six characters (Fig. S1), three of which are unique and not contradicted (66:1, gonocoxite with multiple parabasal setae; 67:1, parabasal seta(e) of gonocoxite differentiated; and 69:1, parabasal setae borne on a small protuberance or swelling). However, despite this, the clade receives zero GC support and so does not appear in the collapsed tree (Fig. 3). The inclusion of Bironella within genus Anopheles contradicts the results of other analyses based on molecular and morphological data (Besansky \& Fahey 1997; Foley et al. 1998; Harbach \& Kitching 1998; Krzywinski et al. 2001b), but is consistent with the analyses of Sallum et al. (2002) based on ribosomal (18S, 28S) and mitochondrial (COI, COII) DNA sequences that support the paraphyly of genus Anopheles relative to Bironella.

Relationships of the informal group taxa of the clade are delineated in Figure 6. As is the case with subgenus Cellia, the relationships within subgenus Anopheles are much less resolved than in our previous study (Fig. S2). The Angusticorn and Laticorn Sections, the Anopheles and Cycloleppteron Series of the former and the Myzorhynchus Series of the latter are not monophyletic. The Anopheles Series is not monophyletic because it excludes $A n$. punctipennis (as previously), and two species Groups (Aitkenii and Stigmaticus) fall within a paraphyletic relationship to a clade comprised of Stethomyia $+(\text { Baimaia }+ \text { Bironella })_{2}$ the three of which were basal and paraphyletic to all other Anopheles in the previous study (Fig.

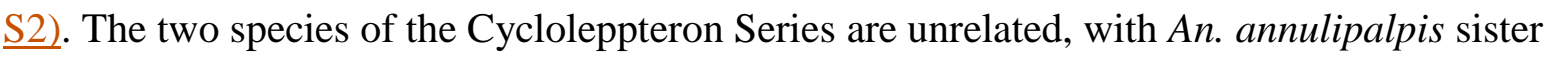
to a clade comprised of the Coustani + Hyrcanus Groups. Only the Lophoscelomyia Series of the Angusticorn Section and the Arribalzagia Series of the Laticorn Section are recovered as monophyletic groups.

When Reid \& Knight (1961) proposed the classification of subgenus Anopheles, they listed the informal group taxa in order from the Laticorn Section, with the Christya, 
Arribalzagia and Myzorhynchus Series, and the Angusticorn Section, with the Cycloleppteron, Lophoscelomyia and Anopheles Series. This order was thought to reflect relationships "reasonably well", with the Christya Series being the "more primitive" and Anopheles Series the "more advanced". As noted above, the results of the present study support the "more primitive" position of the Christya Series, but polyphyletic arrangement of the Anopheles Series (Fig. 6) fairly convincingly shows that it does not include the "more advanced" species of the subgenus. The Anopheles Series was also found to be polyphyletic in previous studies, with its members interspersed in a complexity of inter-group relationships (Sallum et al. 2000) and with Bironella, Stethomyia and Lophopodomyia interspersed within it (Harbach \& Kitching 2005). The present results differ in that part of the Anopheles Series is recovered in a sister relationship to a terminal clade comprised of Stethomyia + (Baimaia + Bironella) (Fig. 6). It is interesting to note that collapsing those groups in Fig. 2 with a GC value of less than 1 does not affect the relationships within the clade comprising An. aitkenii $+($ An. corethroides $+($ Stethomyia $+($ Baimaia + Bironella $)))$ (Fig. 3). Sallum et al. (2000) recovered Bironella and Stethomyia as monophyletic sister groups in the absence of Baimaia, and concluded that they should be classified as informal groups within genus Anopheles rather than generic-level taxa. In their subsequent molecular study (Sallum et al. 2002), Bironella was placed as sister to Lophopodomyia in a clade that also included Nyssorhynchus and Kerteszia, and suggested that Bironella might be treated as a subgenus of Anopheles. Although originally introduced as a genus (Theobald 1905), Bironella was treated as a subgenus of Anopheles by Christophers (1924) and some other authors between 1924 and 1938 (Marks et al. 1963). Belkin (1962) considered Bironella to be an ancient group that shares a number of features with subgenus Anopheles, notably the absence of cibarial armature in adult females and the position and development of seta 1-A on the antennae of larvae. The placement of Bi. gracilis basal to An. atroparvus (subgenus 
Anopheles) + An. gambiae (subgenus Cellia) in the Bayesian likelihood tree of Reidenbach et al. (2009) derived from a data set consisting of six nuclear genes seems to support Belkin's contention; however, the monophyly of genus Anopheles was not supported in all of their analyses - the relationship of Bi. gracilis + An. atroparvus was also recovered. Considering the discordant relationships observed in the phylogenetic studies conducted thus far, it is not possible to determine objectively whether Bironella should retain generic status or be classified as a subgenus or species group within Anopheles. If the relationships indicated in Fig. 3 prove to be correct and Bironella is deemed to warrant generic status, then firmly established monophyletic lineages within the current concept of subgenus Anopheles may also require generic status. Two such groups are the Arribalzagia and Lophoscelomyia Series, both of which were originally established as genera (Arribalzagia Theobald, 1903a and Lophoscelomyia Theobald, 1904). Species of the Arribalzagia Series are the only anophelines that have the subcostal vein of the wing ending in an isolated dark spot distal to the sector dark spot (Wilkerson \& Peyton 1990), which in addition to the six homoplastic characters shown in Fig. S1, confirms the monophyly of the series (Fig. 3: GC = 53). The monophyly of the Lophoscelomyia Series is strongly supported (Fig. 3: $\mathrm{GC}=94$ ) by a combination of five homoplastic characters (Fig. S1). Sallum et al. (2000), and-Harbach \& Kitching (2005) and Collucci \& Sallum (2007) also found strong support for the monophyly of this group. Adults of the Lophoscelomyia Series are distinctive in having white scales on the coxae, dark-scaled legs, and dorsal and ventral scales on the last abdominal segment. Males lack leaflets of the aedeagus.

Collucci \& Sallum (2007) erroneously stated that in contrast "to the hypothesis of Harbach and Kitching (2005), the monophyly of the Cycloleppteron Series" was not supported by the results of their study. However, the Cycloleppteron Series was only represented by An. grabhamii in the study of Harbach \& Kitching, and monophyly cannot be 
demonstrated from only a single included taxon. As in the present study, Collucci \& Sallum found that An. annulipalpis and An. grabhamii are unrelated and the Cycloleppteron Series is a monobasic category that only includes the latter species. Whereas the two species were recovered in a paraphyletic relationship basal to a clade comprised of species of the Arribalzagia, Christya and Myzorhynchus Series in the implied weighting analysis of Collucci \& Sallum, the results of the present study suggest that the two species are more distantly related, with An. annulipalpis sister to a terminal clade comprised of the Coustani and Hyrcanus Groups (Fig. 6). The association of An. annulipalpis with those groups of species is fairly well supported $(\mathrm{GC}=38)$ by three homoplastic characters (Fig. S1). The Coustani-Hyrcanus lineage comprises a homogeneous assemblage of species that are distinguished by the unique presence of lateral patches of scales on the clypeus (Fig. S1, eh.character 6:1). Clypeal scales are absence in An. annulipalpis. In the absence of further information, it is not possible to assign An. annulipalpis to an existing taxonomic group, so for the time being it is retained in the Angusticorn Section as an unplaced species.

The terminal clade consisting of An. aitkenii $+($ An. corethroides $+($ Stethomyia + $($ Baimaia + Bironella $)))$ sits in stark contrast to the relationships of these taxa at the base of genus Anopheles in the phylogeny of Harbach \& Kitching (2005), expressed parenthetically as Baimaia $+($ Bironella $+($ Stethomyia $+($ An. corethroides $+($ all other Anopheles $))))$, with An. algeriensis basal to three other species (An. aitkenii, An. judithae and An. sintonoides) in a clade that is sister to the remaining Anopheles species. The placement of these taxa at opposite ends of the Anopheles topologies is perhaps not as important as their apparent phyletic associations. The Oriental An. aitkenii, unlike the Palaearctic An. algeriensis, which resembles species of the Australian Stigmaticus Group, represented in the analyses by An. corethroides, in having a single parabasal seta, has multiple parabasal setae; this is perhaps why its placement as the sister to An. corethroides $+($ Stethomyia $+($ Baimaia + Bironella $))$ is 
only weakly supported by a GC value of 5 (Fig. 3). The larger sister group is supported by three characters (Fig. S1), one of which is unique and not contradicted (140:1, ventral ramus (sclerotized) reaching the ventral edge of the labiohypopharynx) and a GC support of 23 or 24. The monophyly of subgenus Stethomyia and that of genus Bironella are very strongly supported (GCs $=100$ and 97 respectively) whereas the sister relationship of Baimaia and Bironella is only weakly supported by two homoplastic characters and a GC support of 25 (Fig. 3). If Bironella, Baimaia and Stethomyia are to retain their generic/subgeneric status, then it would seem appropriate to afford the same rank to the Stigmaticus Group.

The results of the present analyses do not agree with the conclusions of Krzywinski et al. (2001a, b), Sallum et al. (2002), Collucci \& Sallum (2003) and Neafsey et al. (2015) that subgenus Anopheles is monophyletic. Sallum et al. (2000), who concluded likewise, included 64 species of Anopheles in their analyses of morphological data, whereas Sallum et al. (2002) only included 32 species in their molecular analyses. The molecular phylogenetic studies of Krzywinski et al. were based on only five species representing the Anopheles (Maculipennis and Pseudopunctipennis Groups), Arribalzagia and Myzorhynchus (Coustani Group) Series, and those of Neafsey et al. included only two species of the subgenus, representing the Maculipennis and Hyrcanus Groups. We are confident that the inclusion of more species of all currently recognized informal groups of the subgenus will confirm the polyphyly of the subgenus. The inclusion of representatives of other taxonomic groups and additional DNA markers in molecular analyses will show, in agreement with morphological data, that subgenus Anopheles is a polyphyletic assemblage of species.

\section{Evolution of Anopheles}

If An. implexus and the species of Lophopodomyia, Stethomyia, Baimaia and Bironella are ignored, the three large clades shown in Fig. 2 are the same as those constructed from the 
protein sequences of 1,085 single-copy orthologs in the study of Neafsey et al. (2015). The topology is the same, with Nyssorhynchus sister to a clade comprised of Anopheles and Cellia as monophyletic sister taxa: Nyssorhynchus $+($ Anopheles + Cellia $)$. If An. implexus is indeed the most primitive species of Anopheles (see above), then it must have evolved from an ancestor that predates the splitting of the lineage that gave rise 100 million years ago to the ancestral lineages of Nyssorhynchus and Anopheles + Cellia (Neafsey et al. 2015). Although the separation of An. implexus from the other Anopheles is only weakly supported (Fig. 3, GC $=2$ ), we nevertheless feel justified, based on the morphological distinctions and similarities with genus Chagasia noted above, in recognizing it as the monotypic member of a separate subgenus; hence, we hereby resurrect the generic name Christya Theobald, 1903, stat. nov., from synonymy with Anopheles Meigen, 1818 and recognize it as a valid subgenus for this species. Theobald (1903b) originally introduced Christya as a genus with Christya implexa as the only included species. In view of the similarities that An. (Christya) implexus shares with species of Chagasia (see above), it might be appropriate to recognize Christya as a separate genus, but doing so in the absence of molecular data for a greater number of Anopheles species would be premature. If, however, Christya is found to be distinct enough to be afforded generic status, available morphological and molecular evidence (Sallum et al. 2002; Neafsey et al. 2015; present study) suggests that Kerteszia + Nyssorhynchus is the most basal group of Anopheles. This would support the suppositions of Belkin (1962), Krzywinski et al. (2001b) and Harbach \& Kitching (2005). Belkin (1962) hypothesized that anophelines initially differentiated in the American Mediterranean Region; Harbach \& Kitching (1998) suggested a possible New World origin of Anophelinae based on the basal placement of Chagasia relative to Anopheles + Bironella in their phylogeny of mosquito genera; and Krzywinski et al. (2001b) provided support for the South American origin of Anophelinae based on a phylogeny of 16 anopheline species inferred from sequences of two protein- 
coding nuclear genes and the Neotropical distributions of Chagasia and subgenera Anopheles, Lophopodomyia, Nyssorhynchus and Stethomyia of Anopheles. However, an alternate hypothesis was more recently proffered by Harbach (2013), who advanced the theory of Christophers (1933) that the ancestral lineage of Anopheles existed before the breakup of Pangaea and subsequently diversified into the extant subgenera after the separation of the continents. The earlier evolution of the lineages that gave rise to Chagasia (South America) and Christya (Africa) favours the Christophers-Harbach evolutionary scenario, but a great deal more work needs to be done before the origins and genealogical relationships of Anophelinae are known with certainty, and a natural classification of the subfamily can be realized.

\section{Concluding comments}

In conclusion, there seems to be little agreement between the phylogenetic relationships of anopheline mosquitoes gleaned from studies conducted thus far $_{2^{-}}$The results of molecular studies in general do not agree with the results of morphological studies because they are based on fewer and different species. Clearly, the exemplar approach is of limited use for reselving deeper relationships and a natural classification of Anophelinae will not be realized until phylogenetic analyses include both morphological and molecular data for most or all species of the subfamily. For the time being, we must continue to use the current generic, subgeneric and informal group taxa as a framework for analysing species relationships and testing phylogenetic hypotheses.

\section{Acknowledgements}

We are grateful to Gustavo Rossi, Centro de Estudios Parasitológicos y de Vectores (CCT La Plata-CONICET-UNLP), La Plata, Argentina, for providing the impetus for the study. 


\section{References}

Anthony, T. G., Harbach, R. E. \& Kitching, I. J. (1999). Phylogeny of the Pyretophorus Series of Anopheles subgenus Cellia (Diptera: Culicidae). Systematic Entomology, 24, 193-205.

Belkin, J. N. (1962). The mosquitoes of the South Pacific (Diptera, Culicidae) [sic]. Vols 1 and 2. Berkeley and Los Angeles, CA: University of California Press.

Besansky, N. J. \& Fahey, G. T. (1997). Utility of the white gene in estimating phylogenetic relationships among mosquitoes (Diptera: Culicidae). Molecular Biology and Evolution, 14, 442-454.

Bourke, B. P., Foster, P. G., Bergo, E. S., Calado, D. C. \& Sallum, M. A. M. (2010). Phylogenetic relationships among species of Anopheles (Nyssorhynchus) Diptera, Culicidae) based on nuclear and mitochondrial gene sequences. Acta Tropica, 114, 8896.

Christophers, S. R. (1915). The male genitalia of Anopheles. Indian Journal of Medical Research, 3, 371-394 + 6 pls.

Christophers, S. R. (1924). Provisional list and reference catalogue of the Anophelini. Indian Medical Research Memoirs, 3, 1-105.

Christophers, S.R. (1933). The fauna of British India, including Ceylon and Burma. Diptera.Vol. IV. Family Culicidae. Tribe Anophelini. London: Taylor and Francis.

Collucci, E. \& Sallum, M. A. M. (2007). Cladistic analysis of the subgenus Anopheles (Anopheles) Meigen (Diptera: Culicidae) based on morphological characters. Memórias do Instituto Oswaldo Cruz, 102, 277-291.

Edwards, F. W. (1932). Genera Insectorum. Diptera, Fam. Culicidae. Fascicle 194. Brussels: Desmet-Verteneuil. 
Faran, M. E. (1980). Mosquito studies (Diptera, Culicidae) XXXIV. A revision of the Albimanus Section of the subgenus Nyssorhynchus of Anopheles. Contributions of the American Entomological Society, 15(7), 1-215.

Foley, D. H., Bryan, J. H., Yeates, D. \& Saul, A. (1998). Evolution and systematics of Anopheles: insights from a molecular phylogeny of Australasian mosquitoes. Molecular Phylogenetics and Evolution, 9, 262-275.

Forey, P. L. \& Kitching, I. J. (2000). Experiments in coding multistate characters, pp. 54-80. In: Scotland, R. \& Pennington, R. T. (eds), Homology and systematics: coding characters for phylogenetic analysis. London: Taylor and Francis.

Gillies, M. T. \& de Meillon, B. (1968). The Anophelinae of Africa south of the Sahara (Ethiopian Zoogeographical Region). Publications of the South African Institute for Medical Research, 54, 1-343.

Goloboff, P. A. (1993). Estimating character weights during tree search. Cladistics, 9, 83-91. Goloboff, P. (1997). Pee-Wee: a program for parsimony analysis under implied weights. Program and documentation, available at ftp.unt.edu.ar/pub/parsimony.

Goloboff, P. A., Carpenter, J. M., Arias, J. S. \& Esquivel, D. R. M. (2008). Weighting against homoplasy improves phylogenetic analysis of morphological datasets. Cladistics, 24, $1-16$.

Goloboff, P. A., Farris, J. S., Källersjö, M., Oxelman, B., Ramírez, M. J. \& Szumik, C. A. (2003). Improvements to resampling measures of group support. Cladistics, 19, $324-332$.

González-Santillán, E. \& Prendini, L. (2015). Phylogeny of North American vaejovid scorpion subfamily Syntropinae Kraepelin, 1905, based on morphology, mitochondrial and nuclear DNA. Cladistics, 31, (in press). 
Grjebine, A. (1966). Faune de Madagascar. XXII. Insecies Diptéres Culicidae Anophelinae. Paris: Centre National de la Recherche Scientifique, Office de la Recherche Scientifique et Technique Outre-Mer.

Harbach, R. E. (1994). Review of the internal classification of the genus Anopheles (Diptera: Culicidae): the foundation for comparative systematics and phylogenetic research. Bulletin of Entomological Research, 84, 331-342.

Harbach, R. E. (2004). The classification of genus Anopheles (Diptera: Culicidae): a working hypothesis of phylogenetic relationships. Bulletin of Entomological Research, 95, 537553.

Harbach, R. E. (2007). The Culicidae (Diptera): a review of taxonomy, classification and phylogeny, pp. 591-688. In: Zhang, Z.-Q. \& Shear, W. A. (eds), Linnaeus Tercentenary: Progress in Invertebrate Taxonomy. Zootaxa, 1668, 1-766.

Harbach, R. E. (2013). The phylogeny and classification of Anopheles, pp. 3-55. In: Manguin, S. (ed.), Anopheles mosquitoes - New insights into malaria vectors. Rijeka, Croatia: InTech.

Harbach, R. E. \& Howard, T. M. (2009). Review of the genus Chagasia (Diptera: Culicidae: Anophelinae). Zootaxa, 2210, 1-25.

Harbach, R. E. \& Kitching, I. J. (1998). Phylogeny and classification of the Culicidae (Diptera). Systematic Entomology, 23, 327-370.

Harbach, R. E. \& Kitching, I. J. (2005). Reconsideration of anopheline phylogeny (Diptera: Culicidae: Anophelinae) based on morphological data. Systematics and Biodiversity, 3, $345-374$.

Krzywinski, J., Wilkerson, R. C. \& Besansky, N. (2001a). Evolution of mitochondrial and ribosomal gene sequences in Anophelinae (Diptera: Culicidae): implications for phylogeny reconstruction. Molecular Phylogenetics and Evolution, 18, 479-487. 
Krzywinski, J., Wilkerson, R. C. \& Besansky, N. (2001b). Toward understanding Anophelinae (Diptera, Culicidae) phylogeny: insights from nuclear single copy genes and the weight of evidence. Systematic Biology, 50, 540-556.

Marks, E. N., Mackerras, I. M., Lee, D. J. \& Iyengar, M. O. T. (1963). Bironella gracilis Theobald, 1905 (Insecta, Diptera): proposed validation under the plenary powers. Bulletin of Zoological Nomenclature, 20, 206-209.

Mohanty, A., Swain, S., Kar, S. K. \& Hazra, R. K. (2009). Analysis of the phylogenetic relationship of Anopheles species, subgenus Cellia (Diptera: Culicidae) and using it to define the relationship of morphologically similar species. Infection, Genetics and Evolution, 9, 1204-1224.

Neafsey, D. E. et al. [120 authors] (2015). Highly evolvable malaria vectors: The genomes of 16 Anopheles mosquitoes. Science, 347: DOI 10.1126/science.1258522.

Nixon, K. C. (1999-2002). Winclada version 1.0000, published by the author. Ithaca, New York. Available at http://www.cladistics.org.

\section{Norris, L.C. \& Norris, D.E. 2015. Phylogeny of anopheline (Diptera: Culicidae) species in} southern Africa, based on nuclear and mitochondrial genes. Journal of Vector

\section{Ecoloogy, 40, 16-27.}

Reid, J. A. 1968. Anopheline mosquitoes of Malaya and Borneo. Studies from the Institute for Medical Research Malaya, 31, 1-520.

Reid, J. A. \& Knight, K. L. (1961). Classification within the subgenus Anopheles (Diptera, Culicidae). Annals of Tropical Medicine and Parasitology, 55, 474-488.

Reidenbach, K. R., Cook, S., Bertone, M. A., Harbach, R. E., Wiegmann, B. M. \& Besansky, N. J. (2009). Phylogenetic analysis and temporal diversification of mosquitoes (Diptera: Culicidae) based on nuclear genes and morphology. BMC Evolutionary Biology, 9, 298. 
Reinert, J. F., Harbach, R. E. \& Kitching, I. J. (2009). Phylogeny and classification of tribe Aedini (Diptera: Culicidae). Zoological Journal of the Linnean Society, 157, 700-794+ 2 online appendices.

Sallum, M. A. M., Foster, P. G., Li, C., Sithiprasasna, R. \& Wilkerson, R. C. (2007). Phylogeny of the Leucosphyrus Group of Anopheles (Cellia) (Diptera: Culicidae) based on mitochondrial gene sequences. Annals of the Entomological Society of America, $100,27-35$.

Sallum, M. A. M., Schultz, T. R., Foster, P. G., Aronstein, K., Wirtz, R. A. \& Wilkerson, R. C. (2002). Phylogeny of Anophelinae (Diptera: Culicidae) based on nuclear ribosomal and mitochondrial DNA sequences. Systematic Entomology, 27, 361-382.

Sallum, M. A. M., Schultz, T. R. \& Wilkerson, R. C. (2000). Phylogeny of Anophelinae (Diptera Culicidae) based on morphological characters. Annals of the Entomological Society of America, 93, 745-775.

Sereno, P. C. (2007). Logical basis for morphological characters in phylogenetics. Cladistics, $23,565-587$.

Theobald, F. V. (1903a). A monograph of the Culicidae or mosquitoes. Volume 3. London: British Museum (Natural History).

Theobald, F. V. (1903b). Report on a collection of mosquitoes and other flies from Equatorial East Africa and the Nile provinces of Uganda. Reports of the Sleeping Sickness Commission of the Royal Society, 7, 33-42, map.

Theobald, F. V. (1904). New Culicidae from the Federated Malay States. Entomologist, 37, $12-15,36-39,77-78,111-113,163-165,211-213,236-239$. 
Theobald, F. V. (1905). A catalogue of the Culicidae in the Hungarian National Museum, with descriptions of new genera and species. Annales Musei Nationalis Hungarici, 3, 61-119, figures legends +4 pls.

Wang, G., Li, C., Guo, X., Xing, D., Dong, Y. \& Zhao, T. (2014). Molecular phylogenetic analysis of the subgenera Anopheles and Cellia (Diptera: Culicidae) based on nuclear ribosomal sequences. African Entomology, 22, 660-669.

Wilkerson, R. C. \& Peyton, E. L. (1990). Standardized nomenclature for the costal wing spots of the genus Anopheles and other spotted-wing mosquitoes (Diptera: Culicidae). Journal of Medical Entomology, 27, 207-224. 


\section{Figure legends}

Fig. 1 Graph showing the summed GC supports at $K$ values $1-33$ and a terminal equal weighted analysis using the groups found in the $K=30$ MPC (see text for details).

Fig. 2 The most parsimonious cladogram found for $K=30$.

Fig. 3 Tree obtained from the $K=30 \mathrm{MPC}$ when $\mathrm{GC}$ values $<1$ are collapsed (only values > 0 are shown). An asterisk denotes values that may be 1 less (e.g. 8 instead of 9 for $A n$. albitarsis + An. braziliensis) in one or more of the 10 replicates (see text for details).

Fig. 4 The Kerteszia + Nyssorhynchus clade of figure 3 with the informal Sections and Series delineated.

Fig. 5 The Cellia clade of figure 3 with the informal Series and species Groups delineated.

Fig. 6 The terminal clade of figure 3 with the informal Sections, Series and species Groups of subgenus Anopheles delineated. 


\section{Supporting information}

Fig. S1. Character support for the clades of the MPC for $K=30$ shown in Fig. 2. Numbers on the branches correspond to the characters and codes listed in Appendix S1 and the data set shown in Appendix S2. Closed circles indicate "unique" character states that can be placed onto the cladogram in only a single position, although they may be interpreted as undergoing subsequent transformation or secondary reversal. Open circles represent homoplastic character states that are placed on more than one branch of the cladogram.

Fig. S2. Phylogeny of subfamily Anophelinae, modified from Harbach \& Kitching (2005), indicating relationships within subgenus Anopheles. Filled circles indicate Bremer support values greater than 0.8 .

Fig. S3. Phylogeny of subgenera Cellia, Kerteszia and Nyssorhynchus, modified from Harbach \& Kitching (2005), indicating relationships within subgenera Cellia and Nyssorhynchus. Filled circles indicate Bremer support values greater than 0.8 .

Appendix S1. Annotated list of morphological characters scored for the two outgroup and 67 ingroup taxa included in the cladistic analyses. The morphological terminology used herein is listed and defined in the Anatomical Glossary of the Mosquito Taxonomic Inventory (http://mosquito-taxonomic-inventory.info/). Numbers in brackets following character numbers are those that Harbach \& Kitching (2005) used in whole or in part for the same characters. See the data matrix in Appendix S2. 
Appendix S2. Data matrix for the 69 taxa and 224 morphological characters included in the cladistic analyses. Missing data are indicated by "?"; characters that could not be scored due to absence of homologous structures are indicated by “_". Polymorphic characters are explicitly coded as such. See Appendix S1 for character descriptions. 